\title{
Internal Iliac Artery Aneurysm Repair with Iliac Branch Endoprothesis
}

Joy Ngai ${ }^{1}$, Gabija Lazaraviciute ${ }^{2}$, Sharon Chan ${ }^{3}$, Kristy Kehoe ${ }^{4}$, Sophie MacDonald ${ }^{5}$, Angharad Jones ${ }^{1}$, Euan Munro ${ }^{6}$, Alisdair Wilson ${ }^{6}$, Athanasios Pantos ${ }^{7}$ and Bryce Renwick ${ }^{6 *}$

${ }^{1}$ Specialty Trainee, General Surgery, Aberdeen Royal Infirmary, UK

${ }^{2}$ Vascular Surgery, Aberdeen Royal Infirmary, UK

${ }^{3}$ Core Surgical Trainee, Vascular Surgery, Aberdeen Royal Infirmary, UK

${ }^{4}$ Foundation Year One Doctor, Vascular Surgery, Aberdeen Royal Infirmary, UK

${ }^{5}$ Physician Associate, Vascular Surgery, Aberdeen Royal Infirmary, UK

${ }^{6}$ Consultant Vascular Surgeon, Vascular Surgery, Aberdeen Royal Infirmary, UK

${ }^{7}$ Consultant Interventional Radiologist, Vascular Surgery, Aberdeen Royal Infirmary, UK

*Corresponding author: Bryce Renwick, Consultant vascular surgeon, Vascular Surgery, Aberdeen Royal Infirmary, UK

Submission: August 01, 2018; Published: August 21, 2018

Abstract

Isolated internal iliac artery aneurysm is a rare condition with limited evidence in the literature to support management strategy. Repair has traditionally been associated with significant morbidity. However advances in endovascular technique offer the potential for new treatment options.

We report our experience of using an off-label device for endovascular repair of an isolated internal iliac artery aneurysm.

\section{Introduction}

The incidence of isolated iliac artery aneurysm has been estimated at $0.3-0.5 \%$ of all intra-abdominal aneurysm, mostly from case series and autopsy data [1]. The rarity of the condition means no standard management approach has been established, with rupture rate and recommendation for treatment still largely based on 35 year old data [2]. The mortality following rupture has Case Report been quoted at $52-58 \%$ [1], and the currently accepted treatment threshold for iliac artery aneurysm at $>3 \mathrm{~mm}[2,3]$. Surgical repair has been the mainstay of treatment, with increasing report of endovascular experience. Intervention may lead to complications of buttock claudication, erectile dysfunction and ischaemic colitis $[1,3]$.

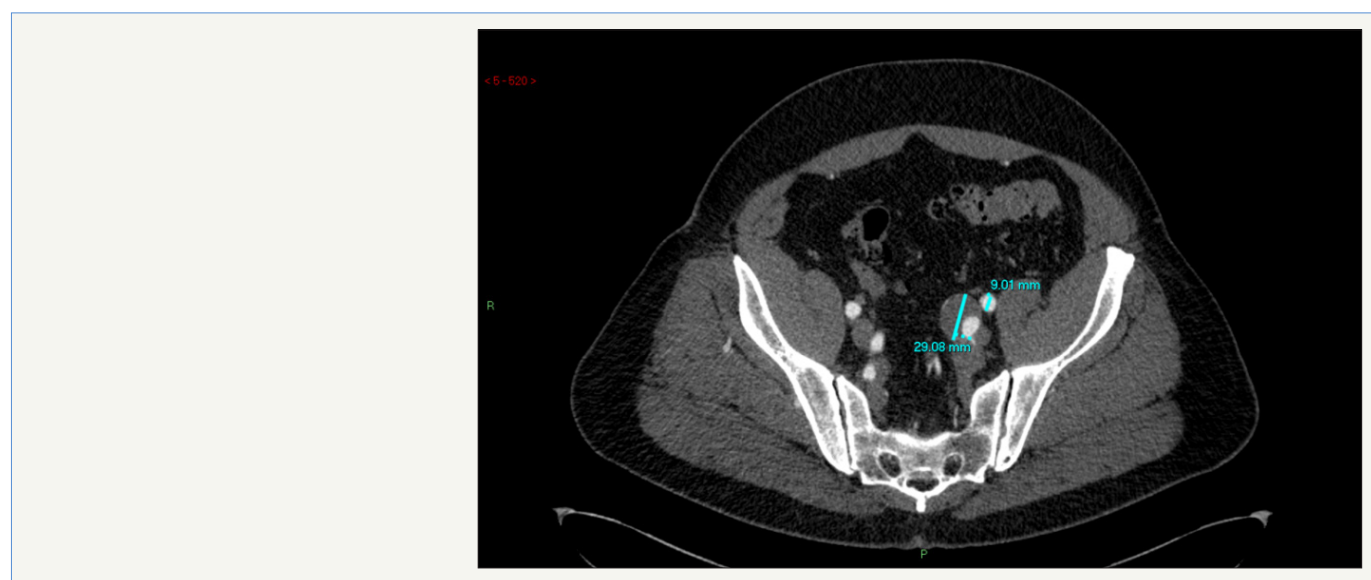

Figure 1: CT Angiogram showing the internal iliac artery aneurysm and external iliac artery on the left. a 
A 59year old gentleman with a past medical history of ulcerative colitis underwent a computed tomography (CT) scan for investigation of pyrexia and left sided abdominal pain under the emergency general surgery team (Figure 1). An incidental finding of an isolated $29 \mathrm{~mm}$ left internal iliac artery aneurysm was noted with a non-aneurysmal abdominal aorta, and he went on to have a formal CT angiogram planning of intervention and discussion at our regional aneurysm multidisciplinary team (MDT) meeting.

Given the accepted current treatment threshold for iliac artery aneurysm at $>3 \mathrm{~mm}[2,3]$ and the significant mortality associated with rupture1, endovascular repair was recommended for this gentleman. Initial discussion with the graft manufacturing company suggested the use of a standard endovascular aneurysm repair (EVAR) graft with exclusion of the left internal iliac artery. However the gentleman works as a security supervisor, which involves a significant amount of walking, and exclusion of the left internal iliac artery would leave him with disabling left buttock claudication that would significantly impact on the quality of his life.
Following further discussion at our MDT meeting and with the graft manufacturer, it was agreed that a branched iliac device could be technically feasible and appropriate in this situation though it would be outside its instruction for use (IFU) [4]. The gentleman was appropriately counselled prior to procedure and understood that should the isolated left iliac branched graft repair be unsuitable due to protrusion into the aortic lumen, he would receive a standard EVAR graft with the implication of life-altering impact.

The procedure was performed endovascularly with bilateral groin percutaneous approach without complication. A GORE EXCLUDER iliac branch endoprosthesis (IBE) $23 \mathrm{~mm} \times 12 \mathrm{~mm} \times$ $10 \mathrm{~cm}$ was placed up to the aortic bifurcation (Figure 2). A proximal branch of the internal iliac artery was embolised with Microvascular Plug (MVP) to prevent a type 2 endo leak. A $9 \mathrm{~mm} \times 5 \mathrm{~cm}$ Viabahn stent was inserted and deployed distally into the posterior division of the left internal iliac arteryand a $16 \mathrm{~mm} \times 10 \mathrm{~mm} \times 7 \mathrm{~cm}$ Gore Excluder extension limb.
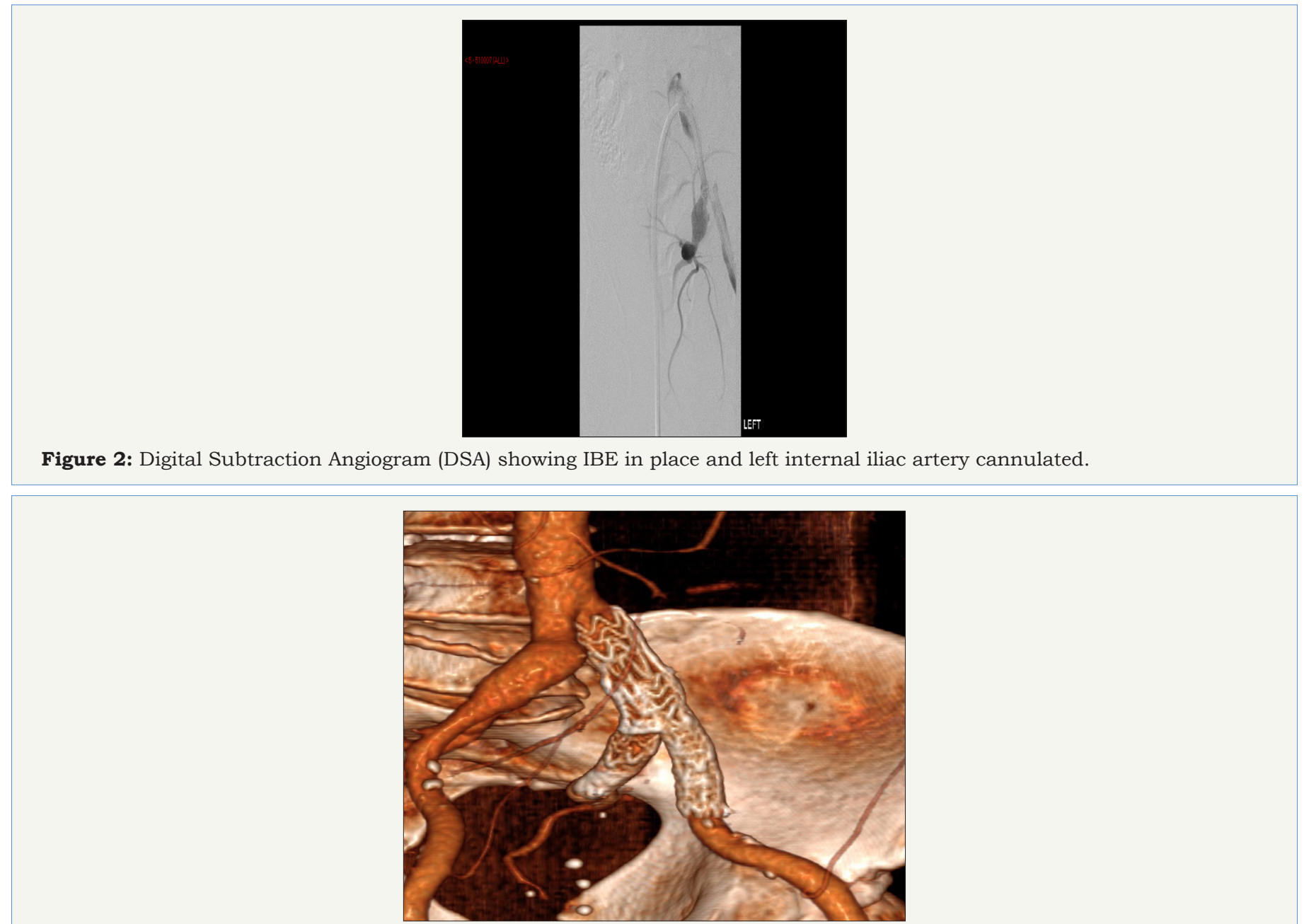

Figure 3: 3D reconstruction of follow-up CT-angiogram demonstrating the stent position and maintained perfusion to the left internal iliac artery.

The gentleman was discharged home one day following his procedure and was commenced on Clopidogrel for secondary prevention. He has since returned to work with no complication.
Follow-up CT at ten weeks shows maintained perfusion to the left internal iliac artery, with further follow-up planned in one year (Figure 3). 


\section{Discussion}

In using an out-of-IFU device, the case underwent rigorous MDT discussions between the consultant vascular surgeon team and consultant interventional radiologist team on multiple occasions in the planning of this gentleman's treatment. He was fully informed regarding the out-of-IFU use of the device, the rationale for the proposal, and what the bail-out strategy would be, with their respective implications as well as the implication for conservative management before consenting to the procedure. It was also important that the implanting team, consisting of both consultant vascular surgeons and interventional radiologists, was familiar with the device, confident that it was technically feasible, and had appropriate time to plan for the procedure as a team.

\section{References}

1. Wilhelm BJ, Sakharpe A, Ibrahim G, Baccaro LM, Fisher J (2014) The 100 -year evolution of the isolated internal iliac artery aneurysm. Ann Vasc Surg 28(4): 1070-1077.

2. McCready RA, Pairolero PC, Gilmore JC, Kazmier FJ, Cherry KJ, et al. (1983) Isolated iliac artery aneurysms. Surgery 93(5): 688-693.

3. Dix FP, Titi M, Al KH (2005) The isolated internal iliac artery aneurysm- a review. Eur J Vasc Endovasc Surg 30(2): 119-129.

4. Gore WL (2018) Instructions for use for: Gore excluder Iliac Branch Endoprosthesis Device.
Creative Commons Attribution 4.0 International License

For possible submissions Click Here

\section{Submit Article}

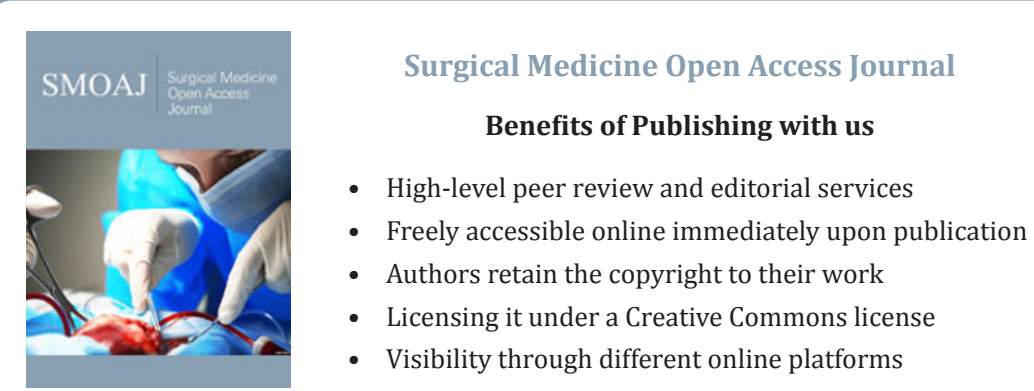

\section{Surgical Medicine Open Access Journal}

\section{Benefits of Publishing with us}

High-level peer review and editorial services

Freely accessible online immediately upon publication

- Visibility through different online platforms 